\title{
Heavy ions: jets and correlations
}

\author{
Gabor VERES* \\ CERN \\ E-mail: gabor.veres@cern.ch
}

Strongly interacting matter can be studied in the laboratory under extremely high energy density and temperature by creating ultrarelativistic heavy-ion collisions. Jets and correlations are invaluable tools to explore properties of this form of matter. Jets are sensitive to the interactions between energetic partons and the medium and to nuclear parton distribution functions, while correlations reveal details about the collective motion of the constituents of this fluid-like phase and can shed light on the role of the initial state properties. A few recent developments and results from the LHC and RHIC accelerators are discussed in this talk.

The European Physical Society Conference on High Energy Physics - EPS-HEP2013

18-24 July 2013

Stockholm, Sweden

${ }^{*}$ Speaker. 


\section{Introduction}

The research of high energy heavy-ion collisions has experienced a remarkable boost since the last edition in this conference series. While the Large Hadron Collider (LHC) has increased the integrated $\mathrm{Pb}+\mathrm{Pb}$ luminosity twenty-fold in the last two years and expanded the scope of the program by collecting $\mathrm{p}+\mathrm{Pb}$ data, the Relativistic Heavy Ion Collider (RHIC) has continued its high statistics $\mathrm{Au}+\mathrm{Au}$ data taking, including steps of the energy scan, and for the first time, $\mathrm{U}+\mathrm{U}$ and $\mathrm{Cu}+\mathrm{Au}$ collisions. The analysis of these high statistics data sets is ongoing, with significant advances of both specific areas discussed in this talk: jets and correlation studies. A wide range of theoretical developments were also triggered by these new results, including various interpretations of novel two-particle correlations, and descriptions of jet modifications in the presence of the strongly interacting medium, parton showers, studies of nuclear parton distribution functions.

Only a limited set of recent and most important jet-related results could be included in this brief summary. Namely, jet energy imbalance studies in $\mathrm{Pb}+\mathrm{Pb}$ and $\mathrm{p}+\mathrm{Pb}$ collisions; jet suppression at high transverse momenta with respect to proton-proton collisions; nuclear modification of jet shape and fragmentation. Correlations are actively studied between charged particles, between jets and photons; jets and hadrons; two jets; just to name a few. In the following, only a brief discussion on the asymmetries of particle and jet production, on two-particle correlations, and especially on long-range correlations in various collision systems will be presented.

Research in heavy-ion physics is primarily related to understanding Quantum Chromodynamics (QCD) and the strong interaction, which - in spite of a simple-looking Lagrangian and a very profound role in Nature - encompasses a wealth of complicated phenomena. Relativistic heavy-ion collisions are suitable experimental tools to study many of these, due to the attainable large energy density and temperature, and the thereby emerging new phases of matter, characterized by partonic degrees of freedom. The interplay between perturbative and non-perturbative processes makes this subject intriguing and challenging both theoretically and experimentally. Experiments with ultrarelativistic ion collisions may enlighten us about certain aspects of hadron structure at high energy (saturation, nuclear modifications of the parton distribution functions), and the properties of the created hot and dense medium (thermalization, hydrodynamical behavior, the nature of its building blocks and of the interactions between them).

Heavy-ion collisions occur at a randomly distributed impact parameter. In central collisions most of the nucleons interact, while peripheral (grazing) collisions have spectator and participant nucleons. The collision centrality is characterized by one of three quantities: a) the percentile of the total inelastic hadronic cross section, based on a given quantity that has a monotonic relationship with the impact parameter (i.e. $0-10 \%$ meaning the $10 \%$ most central collisions); b) the number of nucleons participating in the collision (participants, $N_{\text {part }}$ ); c) the number of binary collisions, $N_{\text {coll }}$, between nucleon pairs. While a) is a measurable quantity, the determination of $N_{\text {part }}$ and $N_{\text {coll }}$ also relies on a Glauber model and detector simulation. The number of hard/rare processes, such as the production of very energetic jets and hadrons, electroweak bosons and quarkonium states is expected to be proportional to $N_{\text {coll }}$ in the absence of nuclear effects, thus $N_{\text {coll }}$ is commonly used in comparisons between particle yields measured in $\mathrm{p}+\mathrm{p}$ and $\mathrm{Pb}+\mathrm{Pb}$ collisions.

At the RHIC and LHC accelerators, three large experiments are designed for heavy-ion measurements: STAR [1], PHENIX [2] and ALICE [3]. ALICE and STAR feature large volume time 
projection chambers and are optimized for particle identification, employing virtually all known identification techniques. These experiments have excellent low momentum tracking. ALICE also has a forward muon spectrometer and a low-mass tracker with precise vertexing capabilities, and suited to reconstructing and triggering on jets. PHENIX excels in pion, kaon, proton, electron and photon identification in its central arms, and in muon identification in the muon arms.

ATLAS [4] and CMS [5] are general purpose experiments at the LHC, and they are powerful tools to study ion collisions, especially jets (due to their excellent calorimetry with large angular coverage, $|\eta|<5$ ) and correlations (due to their tracking systems, also extended in the $|\eta|<2.5$ region). Jets can be reconstructed combining the tracks and calorimeter signals, they have excellent muon detection capabilities and track momentum resolution up to hundreds of $\mathrm{GeV} / \mathrm{c}$ transverse momenta, and equipped with forward calorimeters (ZDC, CASTOR, HF). Their flexible multi-level trigger systems designed for very high luminosities are invaluable for the success of jet and correlation studies. The integrated luminosity of relevant data sets collected by these LHC experiments amounts to about $160 \mu \mathrm{b}^{-1}$ for $\mathrm{Pb}+\mathrm{Pb}$ collisions at $\sqrt{s_{\mathrm{NN}}}=2.76 \mathrm{TeV}, 30 \mathrm{nb}^{-1}$ for $\mathrm{p}+\mathrm{Pb}$ collisions at $\sqrt{s_{\mathrm{NN}}}=5.02 \mathrm{TeV}$ and $5 \mathrm{pb}^{-1}$ for $\mathrm{p}+\mathrm{p}$ collisions at $\sqrt{s}=2.76 \mathrm{TeV}$.

\section{Jets in heavy-ion physics}

The lifetime of the hot and dense medium created in a heavy-ion collision is too short to apply any external source to irradiate it in order to obtain tomographic information. Instead, internal sources, 'probes' that are created in the collision itself are commonly employed for this purpose. High- $p_{T}$ jets are a prominent choice, since they have been extensively studied both theoretically and experimentally (in $e^{+} e^{-}$and $\mathrm{p}+\mathrm{p}(\overline{\mathrm{p}})$ colliders), and represent color-charged objects (quarks, gluons) that strongly interact with the mentioned medium. Modification of the yields (cross sections), shapes, fragmentation, and correlations of jets with respect to those quantities measured in elementary particle collisions are related to the properties of the strongly interacting matter under these extreme conditions. The first observations of the very sizeable reduction in the jet (hadron) yields at high $p_{T}$ in heavy-ion collisions when compared to the $\mathrm{p}+\mathrm{p}$ baseline, was made a decade ago at RHIC by the STAR and PHENIX experiments (confirmed by PHOBOS and BRAHMS), and named 'jet quenching'. At the LHC the jet cross sections are higher, and the separation of jets from the background of the abundant 'soft' processes also pose less experimental complications.

The most frequently used prescription to combine jet components at the LHC is the anti- $k_{T}$ algorithm [6], with a rather small radius parameter between 0.2 and 0.5 , to minimize the fluctuations of the underlying event background. An example to subtract this background is an iterative procedure, where the background is calculated in constant pseudorapidity $(\eta)$ slices, and subtracted from the energy density measured in the $\eta-\phi$ plane, where $\phi$ is the azimuthal angle around the beam-line. In the second step, jets are reconstructed, and the background is recalculated with the exclusion of the jets, and the subtraction is repeated. In the following, the jets are reconstructed again, taking into account the refined background subtraction [7].

The first and most spectacular manifestation of the parton energy loss in the strongly interacting medium was a pronounced dijet energy imbalance in central $\mathrm{Pb}+\mathrm{Pb}$ collisions $[8,9]$. At the same time, the jet partners are observed to remain back-to-back in $\phi$, suffering no more angular decorrelation than dijets in $p+p$ collisions [9]. These initial results have not been sufficient to clearly 

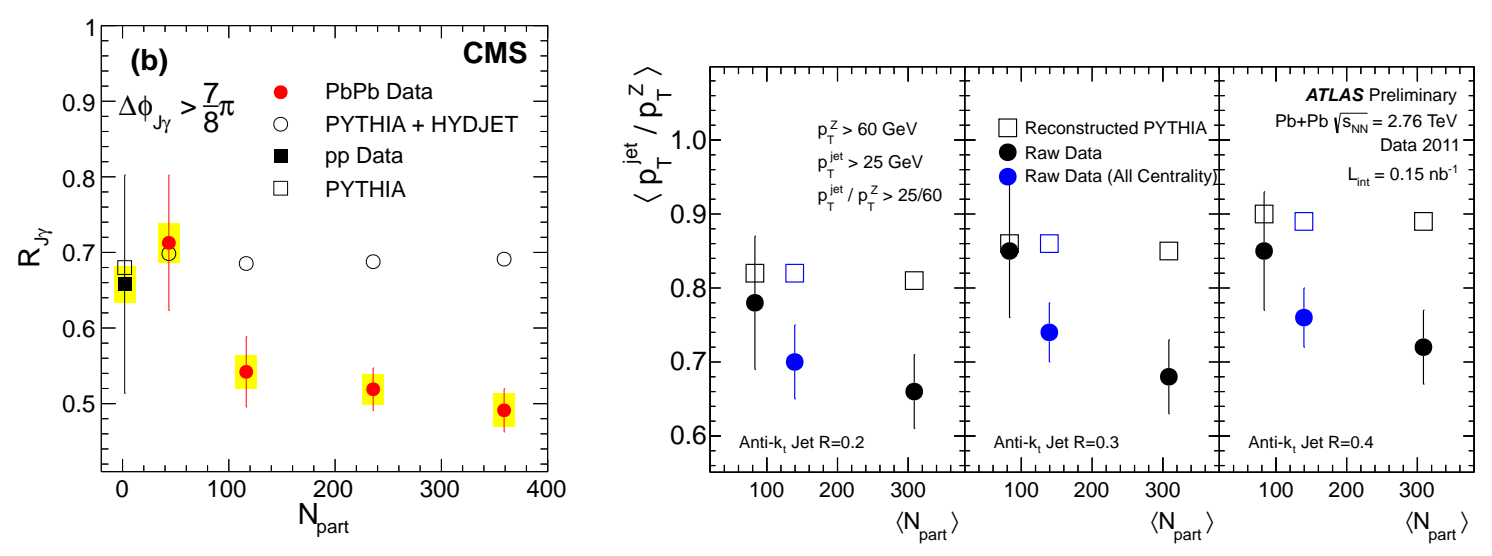

Figure 1: Left panel: Average fraction of isolated photons with an associated jet above $30 \mathrm{GeV} / \mathrm{c}, R_{J \gamma}$, as a function of $N_{\text {part }}$. The yellow boxes indicate point-to-point systematic uncertainties and the error bars denote the statistical uncertainty [13]. Right panel: The mean $p_{\mathrm{T}}^{\mathrm{jet}} / p_{\mathrm{T}}^{Z}$ values for different jet cone sizes, compared with Pyтнia $Z$ boson + jet simulated events, as a function of $\left\langle N_{\text {part }}\right\rangle$. Bars represent statistical uncertainties. The blue points refer to $0-80 \%$ centrality [14].

distinguish between various possible scenarios for parton energy loss, such as soft collinear radiation (GLV and others [10]), hard radiation (PYTHIA-inspired models, modified splitting functions [11]) and large-angle soft radiation (QGP heating, AdS/CFT [12]), and more detailed experimental investigations have continued.

In order to 'calibrate' the jet (parton) energy loss, it is instructive to correlate colorless particles, such as high energy photons or $Z$ bosons, with jets. The photon or $Z$ tag provides the direction and $p_{T}$ of the initial quark to first order. Besides the $\gamma$-jet energy imbalance, the fraction of high- $p_{T}$ photons that had a jet in the azimuthally opposite direction $(\Delta \phi>7 \pi / 8)$ above a certain $p_{T}$ threshold was found to be significantly - by about $20 \%$ - smaller in the most central $\mathrm{Pb}+\mathrm{Pb}$ collisions than in $p+p$ collisions [13], as shown on the left panel of Fig. 1. Both $\gamma$-jet and $Z$-jet events exhibit a decreasing trend of the $p_{T}^{\text {jet }} / p_{T}^{\gamma(Z)}$ ratio as a function of $N_{\text {part }}$, indicating that jets lose about 15-20\% of their $p_{T}$ in central heavy-ion collisions compared to the same ratio for the $\mathrm{p}+\mathrm{p}$ reference $[13,14]$ (right panel of Fig. 1).

The centrality-dependence of the suppression of the inclusive single jet yield can be expressed either by the quantity $R_{C P}$, which is the ratio between the jet $p_{T}$ distributions in central and peripheral collisions (each normalized by $\left\langle N_{\text {coll }}\right\rangle$ in the respective centrality bin), or by $R_{A A}$, which has a similar definition except that peripheral ion collisions are replaced by $\mathrm{p}+\mathrm{p}$ collisions. A continuous evolution of the ratio $R_{C P}$ is found as a function of $N_{\text {part }}$ for charged particle jets by ALICE [17] and for full jets by ATLAS [18]. The ratio $R_{A A}$ for full jets was measured by CMS [15], and a suppression of the jet yield by a factor of two was obtained in the $p_{T}>100 \mathrm{GeV} / \mathrm{c}$ range for central collisions, as can be seen on the left panel of Fig. 2. The ALICE experiment has also measured the $R_{A A}$ for jets in $\mathrm{Pb}+\mathrm{Pb}$ collisions [16], which is found to be in qualitative agreement with the nuclear modification factors of charged hadrons (right panel of Fig. 2).

The parton propagation in the medium can give rise to modifications of the jet shape and 

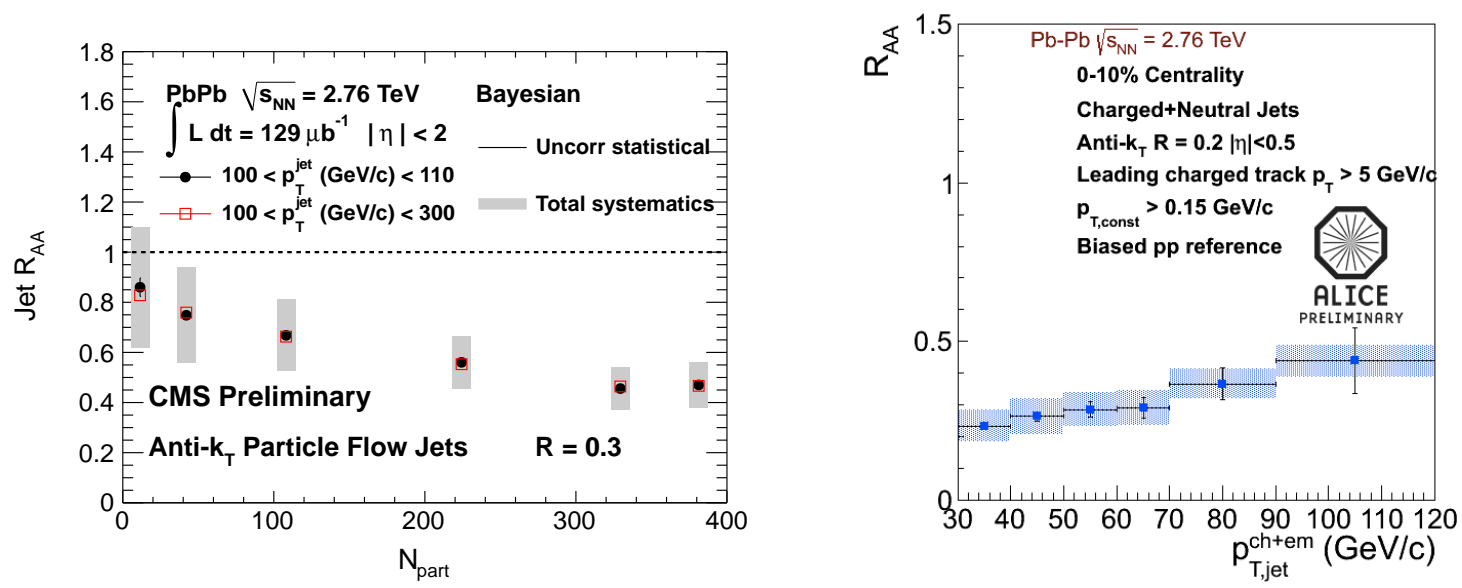

Figure 2: Left panel: Bayesian unfolded jet $R_{A A}$ for anti- $k_{T}$ jets of $\mathrm{R}=0.3$ as a function of $N_{\text {part }}$. Closed circles represent the jet $R_{A A}$ for $100<p_{T}<110 \mathrm{GeV} / \mathrm{c}$, and open boxes the jet $R_{A A}$ for $100<p_{T}<300 \mathrm{GeV} / \mathrm{c}$. Vertical lines represent the uncorrelated statistical uncertainty and the wide grey bands represent the systematic uncertainty combined with the uncertainty from $T_{A A}$ [15]. Right panel: The $R_{A A}$ in the $10 \%$ most central events [16]. The statistical and systematic uncertainties from the pp and $\mathrm{Pb}+\mathrm{Pb}$ analyses are added in quadrature. The combined systematic uncertainties are shown as dotted boxes.

fragmentation pattern, which may serve to clarify the role of various mechanisms in the parton energy loss. The simplest approach to measure jet shapes is to form the ratio of jet cross sections with different radius parameters. Such ratio was found to be comparable between $p+p$, central and peripheral $\mathrm{Pb}+\mathrm{Pb}$ collisions by the ALICE experiment for charged particle jets within systematic uncertainties in the $40<p_{T}<110 \mathrm{GeV} / \mathrm{c}$ range [19]. A more sensitive observable to small deviations of the jet structure is the distribution of transverse momentum carried by charged particles as a function of the distance $r=\sqrt{\Delta \eta^{2}+\Delta \phi^{2}}$ from the jet axis. A modest but significant excess of this quantity was observed in central $\mathrm{Pb}+\mathrm{Pb}$ collisions, compared to peripheral and $\mathrm{p}+\mathrm{p}$ collisions [20]. The number distribution of particles is also increased by about one particle per jet on average, at low $p_{T}$ in central $\mathrm{Pb}+\mathrm{Pb}$ collisions, as shown by the fragmentation pattern results of ATLAS and CMS $[21,22]$. A low- $p_{T}$ excess at large angles with respect to the jet axis was also found by PHENIX in $\gamma$-hadron correlations [23]. Studies of low- $p_{T}$ jets and associated hadrons are an essential ingredient to study jet modification, which are however complicated by the underlying event background. The analysis of high-statistics Au+Au datasets at RHIC suggests a jet broadening at low $p_{T}$, compared to $\mathrm{p}+\mathrm{p}$ collisions, deduced from correlations between near-side jets and away-side charged hadrons (as a means to mitigate the bias on the away side).

In summary, the experimental evidence shows that partons (jets) lose a significant amount of energy in the strongly interacting medium, while jet pairs remain in tight azimuthal correlation. Jet fragmentation patterns for leading hadrons are not modified (relative to the jet $p_{T}$ ) as opposed to low $p_{T}$, where an excess energy and number of fragments is observed, preferentially at large angles with respect to the jet axis. In the meantime, the theoretical treatment of the the subject advances from treatments of parton energy loss and leading hadrons towards studies of modification of parton showers and jets. Since jet production is a weakly coupled phenomenon, and the medium 

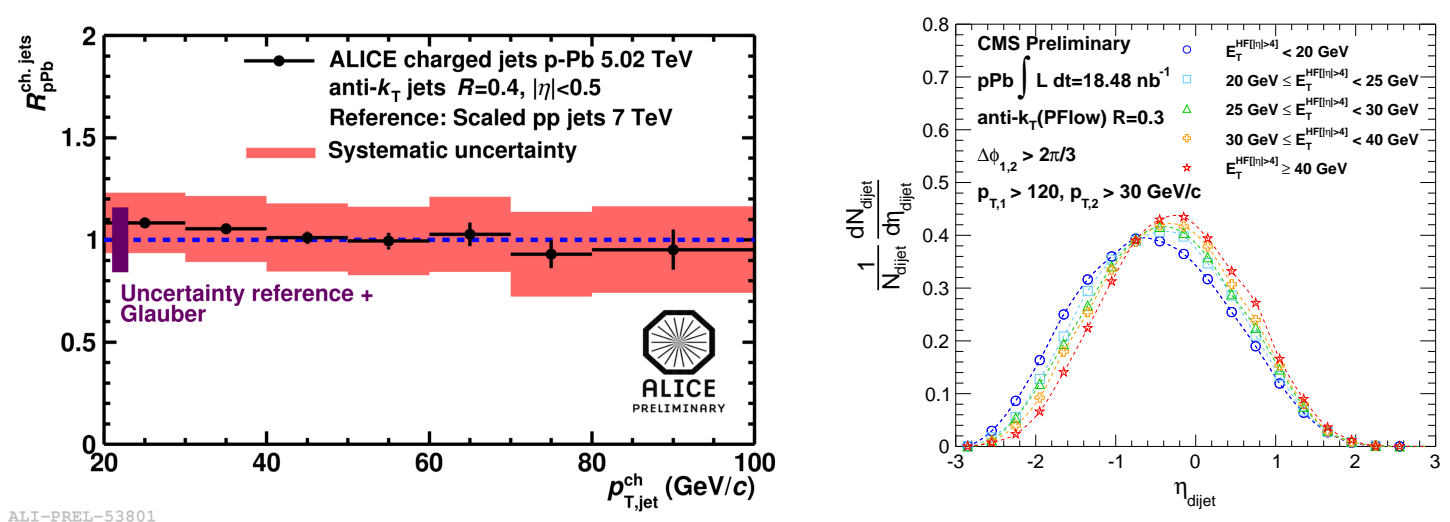

Figure 3: Left panel: Nuclear modification factors, $R_{p P b}$, for charged particle jets in $\mathrm{p}+\mathrm{Pb}$ collisions at $\sqrt{s_{N N}}=5.02 \mathrm{TeV}$ reconstructed with the anti- $k_{T}$ algorithm [25]. Right panel: Dijet pseudorapidity distributions in five HF activity classes. The distributions are normalized by the number of selected dijet events [26].

shows signs of a strongly coupled fluid, the appropriate theoretical approach should probably be a hybrid and sufficiently complex one. First-principle lattice QCD calculations are also underway to study the jet quenching parameter [24]. Correlation measurements already play an important role here, since various correlations between photons and hadrons, between jets and hadrons, etc., may differentiate between possible theoretical approaches.

The $\mathrm{p}+\mathrm{Pb}$ collisions at $\sqrt{s_{N N}}=5.02 \mathrm{TeV}$ delivered by the LHC in early 2013 serve as an important point of comparison with heavy-ion and elementary collisions. Charged particle jets in minimum bias $\mathrm{p}+\mathrm{Pb}$ collisions have been shown by the ALICE experiment to have the cross section as expected from $\mathrm{p}+\mathrm{p}$ collisions and $N_{\text {coll }}$ scaling in the $20<p_{T}<100 \mathrm{GeV} / \mathrm{c}$ range, within about $20 \%$ systematic and $15 \%$ additional scale (reference) uncertainty [25], depicted on the left panel of Fig. 3. This is an experimental indication that the jet suppression observed in $\mathrm{Pb}+\mathrm{Pb}$ collisions cannot be explained by initial state effects. Although this result does not directly point to any jet suppression, modification of nuclear PDF in $\mathrm{p}+\mathrm{Pb}$ collisions, other observables may be more sensitive to such effects. The CMS experiment has studied the $\eta$ distribution of dijets, and a systematic and significant shift of the distribution was observed as a function of the forward transverse energy (activity) in the collision. Dijets in central events are shifted more to the direction of the $\mathrm{Pb}$ ion [26], as shown on the right panel of Fig. 3. The shift of the $\eta_{\text {dijet }}$ distribution in minimum bias $\mathrm{p}+\mathrm{Pb}$ collisions is only reproduced by $\mathrm{pQCD}$ calculations if the nuclear modifications of the PDFs are taken into account. The distribution of dijet momentum imbalance, $p_{T, 2} / p_{T, 1}$, is not modified even in central $\mathrm{p}+\mathrm{Pb}$ collisions compared to the $\mathrm{p}+\mathrm{p}$ reference with the present experimental precision [26], although very peripheral $\mathrm{Pb}+\mathrm{Pb}$ events - comparable in forward energy flow - are not expected to show a significant effect either.

\section{Correlation studies with heavy ions}

Generally, correlation measurements are widely employed in heavy-ion physics, starting from the collective motion of the strongly coupled medium to the already mentioned hard processes 

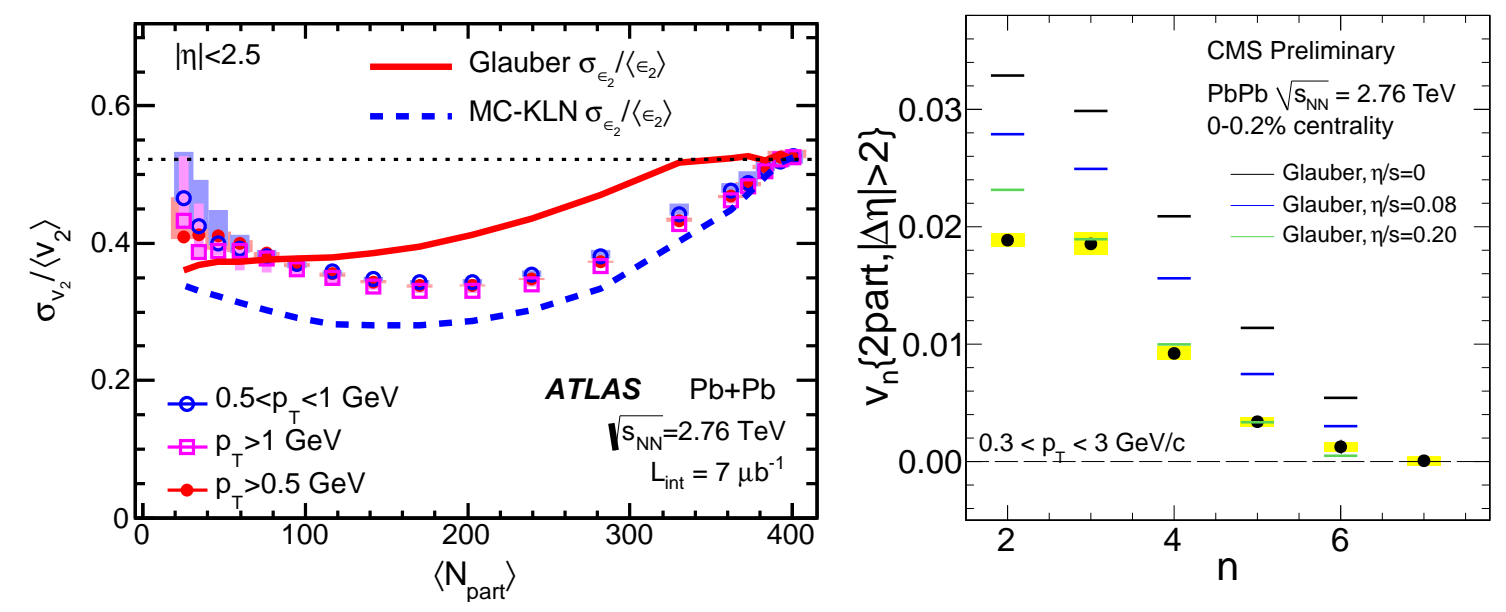

Figure 4: Left panel: $\sigma_{v_{2}} /\left\langle v_{2}\right\rangle$ for three $p_{T}$ ranges together with the total systematic uncertainties [28]. The values of $\sigma_{v_{2}} /\left\langle v_{2}\right\rangle$ are compared with the $\sigma_{\varepsilon_{2}} /\left\langle\varepsilon_{2}\right\rangle$ given by the Glauber model [29] and MC-KLN model [30]. Right panel: Fourier-components, $v_{n}$, measured in ultra-central $\mathrm{Pb}+\mathrm{Pb}$ collisions, compared to models calculations with different $\eta / s$ values [31].

involving jets, electroweak bosons and hadrons. A limited selection of recent developments on correlations will be discussed in this section, concentrating on non-perturbative phenomena.

As it was shown by the pioneering measurements at the SPS and RHIC accelerators, the form of matter created in highly energetic heavy-ion collisions is capable of collective motion, which manifests itself as a correlation between the direction of the motion of produced particles and the impact parameter vector, and consequently, also between pairs of final state particles. The azimuthal asymmetry of the almond-shaped initial collision zone translates into an azimuthal asymmetry of the final state particle distribution through hydrodynamical flow. The distribution of final state particles takes the form of $d N / d \phi \propto 1+\sum_{n=1}^{\infty} 2 v_{n}\left(p_{T}, y\right) \cos [n(\phi-\Psi)]$, and the Fourier coefficients, $v_{n}$, are related to the properties of the strongly interacting fluid, such as shear viscosity over entropy, $\eta / s$. Results on identified particles reveal a quark number scaling of these coefficients, highlighting the role of partonic degrees of freedom. Since asymmetries of single particle distributions are inherited by two-particle distributions, two-particle correlations constitute important and simple experimental means to study these phenomena.

Notably, jets - similarly to charged particles at low $p_{T}$ - are not emitted uniformly either, but for a very different reason: the parton energy loss in the QCD medium depends on the path length traveled, thus the asymmetric overlap zone implies that the $v_{2}$ coefficient of jets will be non-zero, as confirmed by the recent results from ATLAS [27]. Whenever the focus of interest is the collective phenomena of the bulk particles (mostly at low $p_{T}$ ), correlations from jets ('non-flow effects') should be suppressed. A common way to achieve that is to require a large $\Delta \eta$ separation between correlated particles, the small $\Delta \eta$ region being dominated by jets and resonance decays.

The $v_{2}$ coefficient, termed 'elliptic flow' has been measured by ALICE, ATLAS and CMS in great detail, as a function of centrality, $\eta$ [32], particle mass [33] (including open charm [34]), and even its fluctuations event-by-event [28], shown on the left panel of Fig. 4. Higher order 

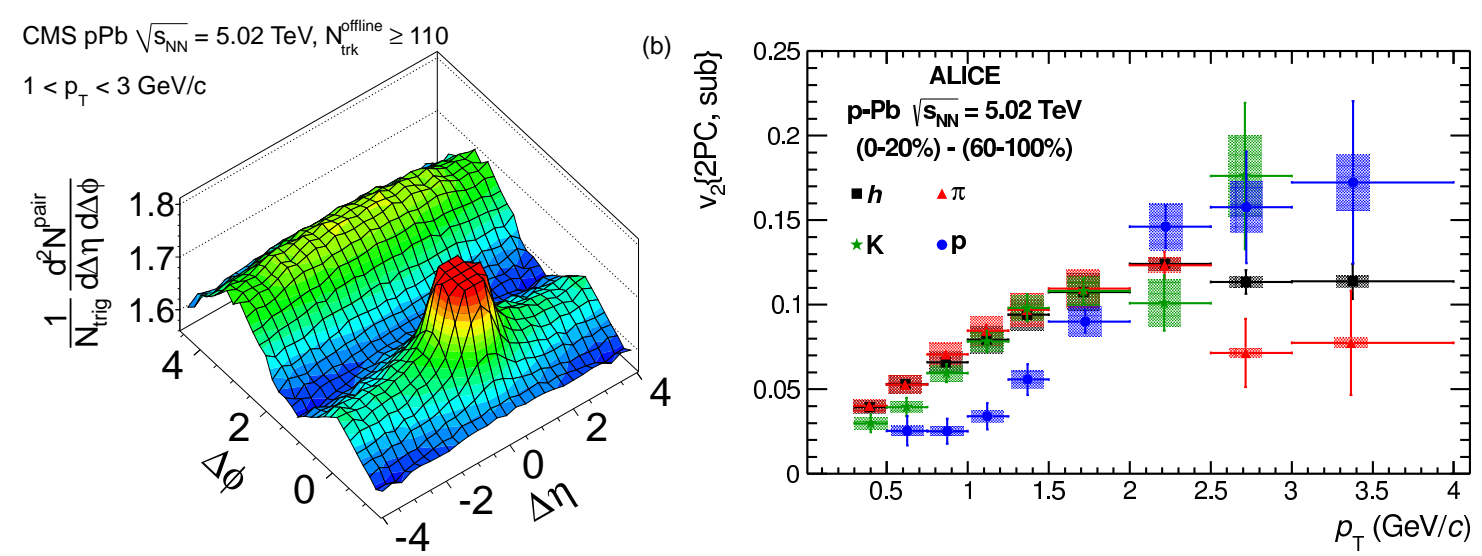

Figure 5: Left panel: 2D two-particle correlation function for $\mathrm{p}+\mathrm{Pb}$ collisions for charged particle pairs with $1<p_{T}<3 \mathrm{GeV} / \mathrm{c}$ and for $N_{\mathrm{trk}}^{\text {offline }} \geq 110$ [38]. Right panel: the second Fourier coefficient for hadrons (black squares), pions (red triangles), kaons (green stars) and protons (blue circles) as a function of $p_{T}$ in central $\mathrm{p}+\mathrm{Pb}$ collisions after subtraction of the correlation from the peripheral multiplicity class. The data is plotted at the average $p_{T}$ for each considered $p_{T}$ interval and particle species under study. Error bars show statistical uncertainties while shaded areas denote systematic uncertainties [44].

coefficients, most importantly $v_{3}$, are related to initial state fluctuations and hydrodynamic properties, and the exploration of the full Fourier-spectrum (two-particle correlation functions) has been also carried out [35]. A unique perspective on hydrodynamic flow is offered by the $v_{n}$ values in ultra-central $\mathrm{Pb}+\mathrm{Pb}$ collisions and their comparison to hydrodynamic calculations, because of their sensitivity to the $\eta / s$ quantity [31], as illustrated on the right panel of Fig. 4.

Perhaps the most striking recent correlation measurement emerged from the analysis of very high multiplicity $\mathrm{p}+\mathrm{p}$ collisions at $7 \mathrm{TeV}$, where a long-range $(|\Delta \eta|>2)$, near-side $(\Delta \phi<1)$ structure (called the 'ridge') was observed by CMS [36], qualitatively similar to that already seen in $\mathrm{Pb}+\mathrm{Pb}$ collisions both at RHIC and at the LHC [37]. A similar correlation structure was discovered in central $\mathrm{p}+\mathrm{Pb}$ collisions as well by CMS [38], as shown on the left panel of Fig. 5. While the interpretation of the ridge in heavy-ion collisions is widely accepted to be a consequence of the fluctuations of the initial geometry and the hydrodynamical evolution, in case of more elementary collisions there are various competing theoretical explanations. Such an expression of hydrodynamical behavior was hardly expected there, and efforts are ongoing to account for the ridge by initial state effects alone, invoking the high-density Color Glass Condensate of the initial state [39]. A similar ridge on the away-side was discovered by ALICE, by subtracting the two-particle correlation function obtained in peripheral $\mathrm{p}+\mathrm{Pb}$ collisions from that measured in central collisions [40]. The Fourier-coefficients $v_{2}$ and $v_{3}$ can also be evaluated from the data, using two-particle correlations or other methods, as a function of multiplicity [41] or event activity, as published by ATLAS [42]. Remarkably, the $v_{3}$ coefficients appear to be of the same magnitude in $\mathrm{p}+\mathrm{Pb}$ and $\mathrm{Pb}+\mathrm{Pb}$ collisions, provided that events with the same charged particle multiplicity are compared [41]. The $v_{2}$ and $v_{3}$ coefficients have also been measured using the high luminosity $\mathrm{d}+\mathrm{Au}$ datasets at RHIC [43], yielding qualitatively similar results.

Identified particles may shed more light on the underlying mechanism of the ridge in $\mathrm{p}+\mathrm{Pb}$ 
collisions, since a characteristic mass-ordering $\left(v_{2}(p)<v_{2}(K)<v_{2}(\pi)\right.$ for $\left.p_{T}<2 \mathrm{GeV} / \mathrm{c}\right)$ was observed in heavy-ion collisions both at RHIC [45] and at the LHC [33]. The $p_{T}$-dependence of the $v_{2}$ coefficient for pions and protons is well described by a hydrodynamical model [46]. Remarkably, a similar mass ordering $\left(v_{2}(p)<v_{2}(\pi) \approx v_{2}(K)\right)$ was observed by the ALICE collaboration also in high-multiplicity $\mathrm{p}+\mathrm{Pb}$ collisions, after subtracting the jet-like correlations using low-multiplicity collisions [44] (right panel of Fig. 5). This result suggests that hydrodynamics may also play an important role already in $\mathrm{p}+\mathrm{Pb}$ collisions at high multiplicity.

\section{Summary}

Today, the Large Hadron Collider has completed two and a half years of successful heavy-ion research and opened a new era, while the productive RHIC program is still at its best. Jets and other hard probes (photons, $W$ and $Z$ bosons, quarkonium states) serve as excellent tools to study the QCD medium under extreme conditions due to their large production cross sections at LHC energies. On the other hand, it has also become possible to study $\mathrm{p}+\mathrm{p}$ and $\mathrm{p}+\mathrm{Pb}$ collisions at very high multiplicities, where several experimental observables and phenomena bears similarities to those in heavy-ion collisions. Various properties of the created medium are studied at a quantitative level with a growing variety of tools, such as parton energy loss via single jets, dijets, $\gamma$-jet and $Z$-jet pairs. Detailed jet shape and fragmentation studies are also emerging. Correlation measurements provide insight into the effects of initial geometry, the hydrodynamical evolution of the system and its physical properties. The continuing LHC program together with the RHIC energy scan, the already collected high statistics datasets and future runs are expected to complement each other towards a more thorough understanding of the rich phenomenology of strongly interacting matter.

Acknowledgments. The author is grateful to CERN and to the Hungarian Scientific Research Fund (OTKA) grants NK 81447 and K 81614 for their support.

\section{References}

[1] STAR Collaboration, STAR detector overview, Nucl.Instrum.Meth. A499 (2003) 624-632.

[2] PHENIX Collaboration, PHENIX detector overview, Nucl.Instrum.Meth. A499 (2003) 469-479.

[3] ALICE Collaboration, The ALICE experiment at the CERN LHC, JINST 3 (2008) S08002.

[4] ATLAS Collaboration, The ATLAS Experiment at the CERN LHC, JINST 3 (2008) S08003.

[5] CMS Collaboration, The CMS experiment at the CERN LHC, JINST 3 (2008) S08004.

[6] M. Cacciari, G. P. Salam, and G. Soyez, The Anti-k(t) jet clustering algorithm, JHEP 0804 (2008) 063, [arXiv: 0802.1189].

[7] O. Kodolova, I. Vardanian, A. Nikitenko, and A. Oulianov, The performance of the jet identification and reconstruction in heavy ions collisions with CMS detector, Eur.Phys.J. C50 (2007) 117-123.

[8] ATLAS Collaboration, Observation of a Centrality-Dependent Dijet Asymmetry in Lead-Lead Collisions at $\sqrt{s_{\mathrm{NN}}}=2.77 \mathrm{TeV}$ with the ATLAS Detector at the LHC, Phys.Rev.Lett. 105 (2010) 252303, [arXiv:1011.6182].

[9] CMS Collaboration, Observation and studies of jet quenching in PbPb collisions at nucleon-nucleon center-of-mass energy = 2.76 TeV, Phys.Rev. C84 (2011) 024906, [arXiv: 1102.1957 ]. 
[10] M. Gyulassy, P. Levai, and I. Vitev, Reaction operator approach to nonAbelian energy loss, Nucl.Phys. $\mathbf{B 5 9 4}$ (2001) 371-419, [nucl-th/ 0006010 ].

[11] N. Armesto, G. Corcella, L. Cunqueiro, and C. A. Salgado, Angular-ordered parton showers with medium-modified splitting functions, JHEP 0911 (2009) 122, [arXiv: 0909. 5118].

[12] E. Iancu, Partons and jets in a strongly-coupled plasma from AdS/CFT, Acta Phys.Polon. B39 (2008) 3213-3280, [arXiv:0812.0500].

[13] CMS Collaboration, Studies of jet quenching using isolated-photon+jet correlations in PbPb and pp collisions at $\sqrt{s_{\mathrm{NN}}}=2.76$ TeV, Phys.Lett. B718 (2013) 773-794, [arXiv:1205.0206].

[14] ATLAS Collaboration, Measurement of momentum imbalance in $Z \rightarrow \ell \ell+j e t$ events in lead-lead collisions at $\sqrt{s_{N N}}=2.76 \mathrm{TeV}$ with the ATLAS detector, Tech. Rep. ATLAS-CONF-2012-119, CERN, Geneva, Aug, 2012.

[15] CMS Collaboration, Nuclear modification factor of high transverse momentum jets in PbPb collisions at $\sqrt{s_{\mathrm{NN}}}=2.76 \mathrm{TeV}$, Tech. Rep. CMS-PAS-HIN-12-004, CERN, Geneva, 2012.

[16] ALICE Collaboration, Full Jet Reconstruction in $2.76 \mathrm{TeV} p$ and Pb-Pb collisions in the ALICE experiment, J.Phys.Conf.Ser. 446 (2013) 012006, [arXiv: 1304.5945 ].

[17] ALICE Collaboration, Measurement of jet spectra with charged particles in $\mathrm{Pb}$-Pb collisions at $\sqrt{s_{N N}}=2.76 \mathrm{TeV}$ with the ALICE detector, Nucl.Phys.A904-905 2013 (2013) 1015c-1018c, [arXiv:1210.8264].

[18] ATLAS Collaboration, Measurement of the jet radius and transverse momentum dependence of inclusive jet suppression in lead-lead collisions at $\sqrt{s_{N N}}=2.76 \mathrm{TeV}$ with the ATLAS detector, Phys.Lett. B719 (2013) 220-241, [arXiv:1208.1967].

[19] ALICE Collaboration, Jet Results in pp and Pb-Pb Collisions at ALICE, arXiv:1306.2747.

[20] CMS Collaboration, Modification of jet shapes in PbPb collisions at $\sqrt{s_{N N}}=2.76 \mathrm{TeV}$, arXiv: 1310.0878 .

[21] CMS Collaboration, Detailed characterization of jets in heavy ion collisions using jet shapes and jet fragmentation functions, Tech. Rep. CMS-PAS-HIN-12-013, CERN, Geneva, 2012.

[22] ATLAS Collaboration, Measurement of inclusive jet charged particle fragmentation functions in $P b+P b$ collisions at $\sqrt{s_{N N}}=2.76 \mathrm{TeV}$ with the ATLAS detector, Tech. Rep. ATLAS-CONF-2012-115, CERN, Geneva, Aug, 2012.

[23] PHENIX Collaboration, Medium modification of jet fragmentation in Au+Au collisions at $\sqrt{s_{N N}}=200 \mathrm{GeV}$ measured in direct photon-hadron correlations, arXiv:1212.3323.

[24] M. Panero, K. Rummukainen, and A. SchÃd'fer, A lattice study of the jet quenching parameter, arXiv: 1307.5850 .

[25] ALICE Collaboration, Charged Jets in Minimum Bias p-Pb Collisions at $\sqrt{s}=5.02$ TeV with ALICE, arXiv:1310.3612.

[26] CMS Collaboration, Study of dijet momentum balance and pseudorapidity distributions in $\mathrm{pPb}$ collisions at $\sqrt{s_{\mathrm{NN}}}=5.02 \mathrm{TeV}$, Tech. Rep. CMS-PAS-HIN-13-001, CERN, Geneva, 2013.

[27] ATLAS Collaboration, Measurement of the Azimuthal Angle Dependence of Inclusive Jet Yields in $\mathrm{PbPb}$ Collisions at $\sqrt{s_{\mathrm{NN}}}=2.76 \mathrm{TeV}$ with the ATLAS detector, arXiv: 1306.6469.

[28] ATLAS Collaboration, Measurement of the distributions of event-by-event flow harmonics in lead-lead collisions at $\sqrt{s_{N N}}=2.76 \mathrm{TeV}$ with the ATLAS detector at the LHC, arXiv: 1305.2942. 
[29] M. L. Miller, K. Reygers, S. J. Sanders, and P. Steinberg, Glauber modeling in high energy nuclear collisions, Ann.Rev.Nucl.Part.Sci. 57 (2007) 205-243, [nucl-ex/ 0701025 ].

[30] H.-J. Drescher, A. Dumitru, A. Hayashigaki, and Y. Nara, The Eccentricity in heavy-ion collisions from color glass condensate initial conditions, Phys.Rev. C74 (2006) 044905, [nucl-th/0605012].

[31] CMS Collaboration, Azimuthal anisotropy harmonics in ultra-central PbPb collisions at $\sqrt{s_{N N}}=2.76 \mathrm{TeV}$, Tech. Rep. CMS-PAS-HIN-12-011, CERN, Geneva, 2012.

[32] CMS Collaboration, Measurement of the elliptic anisotropy of charged particles produced in PbPb collisions at nucleon-nucleon center-of-mass energy = 2.76 TeV, Phys.Rev. C87 (2013) 014902, [arXiv:1204.1409].

[33] ALICE Collaboration, Anisotropic flow of charged hadrons, pions and (anti-)protons measured at high transverse momentum in Pb-Pb collisions at $\sqrt{s_{N N}}=2.76 \mathrm{TeV}$, Phys.Lett.B 719 (2013) 18-28, [arXiv:1205.5761].

[34] ALICE Collaboration, D meson elliptic flow in non-central Pb-Pb collisions at $\sqrt{s_{N N}}=2.76 \mathrm{TeV}$, Phys.Rev.Lett. 111 (2013) 102301, [arXiv:1305.2707].

[35] ALICE Collaboration, Harmonic decomposition of two-particle angular correlations in $\mathrm{Pb}-\mathrm{Pb}$ collisions at $\sqrt{s_{N N}}=2.76$ TeV, Phys.Lett. B708 (2012) 249-264, [arXiv:1109.2501].

[36] CMS Collaboration, Observation of Long-Range Near-Side Angular Correlations in Proton-Proton Collisions at the LHC, JHEP 1009 (2010) 091, [arXiv : 1009 . 4122].

[37] CMS Collaboration, Centrality dependence of dihadron correlations and azimuthal anisotropy harmonics in PbPb collisions at $\sqrt{s_{N N}}=2.76$ TeV, Eur.Phys.J. C72 (2012) 2012, [arXiv:1201.3158].

[38] CMS Collaboration, Observation of long-range near-side angular correlations in proton-lead collisions at the LHC, Phys.Lett. B718 (2013) 795-814, [arXiv: 1210.5482$].$

[39] K. Dusling and R. Venugopalan, Comparison of the Color Glass Condensate to di-hadron correlations in proton-proton and proton-nucleus collisions, Phys.Rev. D87 (2013) 094034, [arXiv:1302.7018].

[40] ALICE Collaboration, Long-range angular correlations on the near and away side in pPb collisions at $\sqrt{s_{\mathrm{NN}}}=5.02$ TeV, Phys.Lett. B719 (2013) 29-41, [arXiv:1212.2001].

[41] CMS Collaboration, Multiplicity and transverse momentum dependence of two- and four-particle correlations in pPb and PbPb collisions, Phys.Lett. B724 (2013) 213-240, [arXiv: 1305.0609 ].

[42] ATLAS Collaboration, Measurement with the ATLAS detector of multi-particle azimuthal correlations in pPb collisions at $\sqrt{s_{\mathrm{NN}}}=5.02 \mathrm{TeV}$, Phys.Lett. B725 (2013) 60-78, [arXiv: 1303.2084 ].

[43] PHENIX Collaboration, Quadrupole anisotropy in dihadron azimuthal correlations in central $d+A u$ collisions at $\sqrt{s_{N N}}=200 \mathrm{GeV}$, Phys.Rev.Lett. (2013) [arXiv:1303.1794].

[44] ALICE Collaboration, Long-range angular correlations of $\pi, K$ and $p$ in $p-P b$ collisions at $\sqrt{s_{N N}}=5.02 \mathrm{TeV}$, arXiv:1307.3237.

[45] STAR Collaboration, Azimuthal anisotropy in Au+Au collisions at $\sqrt{s_{N N}}=200$ GeV, Phys.Rev. C72 (2005) 014904, [nucl-ex/ 0409033 ].

[46] C. Shen, U. Heinz, P. Huovinen, and H. Song, Radial and elliptic flow in Pb+Pb collisions at the Large Hadron Collider from viscous hydrodynamic, Phys.Rev. C84 (2011) 044903, [arXiv:1105.3226]. 